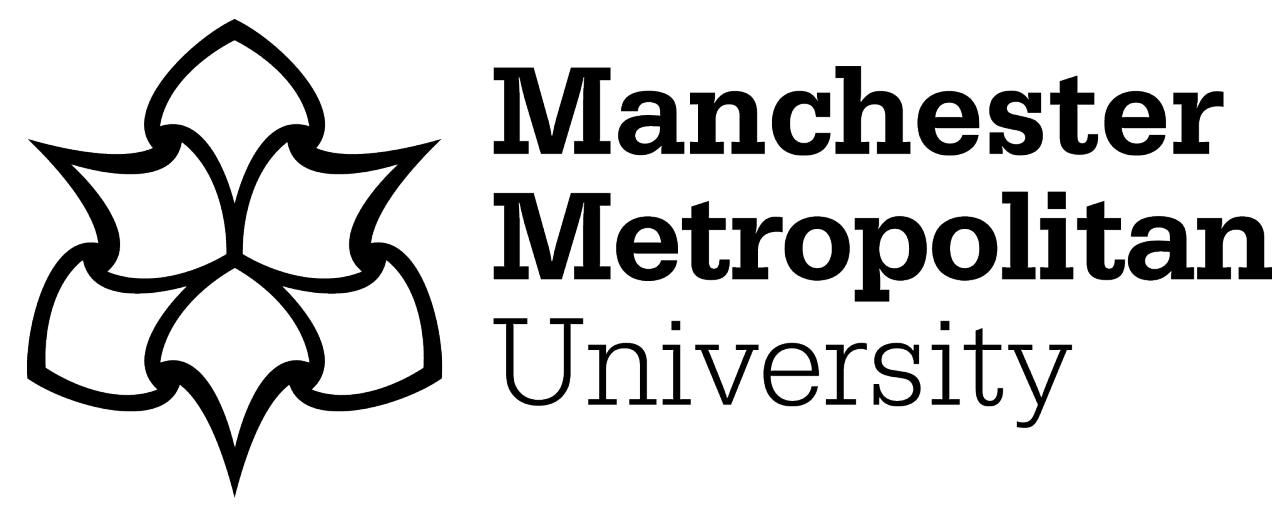

Britten, TK ORCID logoORCID: https://orcid.org/0000-0002-3580-1145, Kemmitt, PD, Halcovitch, NR and Coote, SC (2020) 1,2-Dihydropyridazines as Versatile Synthetic Intermediates. Synlett, 31 (5). pp. 459-462. ISSN 0936-5214

Downloaded from: https://e-space.mmu.ac.uk/625606/

Version: Accepted Version

Publisher: Thieme

DOI: https://doi.org/10.1055/s-0039-1690825

Please cite the published version 


\section{1,2-Dihydropyridazines as Versatile Synthetic Intermediates}

\author{
Thomas K. Brittena \\ Paul D. Kemmittb \\ Nathan R. Halcovitcha \\ Susannah C. Coote*a \\ a Department of Chemistry, Lancaster University, Bailrigg, LA1 \\ 4YB, U.K. \\ ${ }^{\mathrm{b}}$ Medicinal Chemistry, Research and Early Development, \\ Oncology R \& D, AstraZeneca, Cambridge, CB10 1XL, U.K. \\ s.coote@lancaster.ac.uk
}

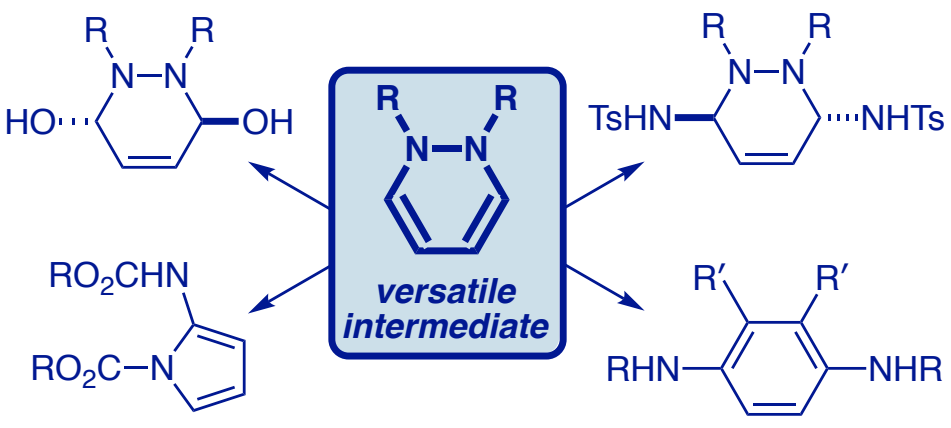

\section{Received:
Accepted:
Published onlin \\ DO}

Abstract The reactivity of 1,2-dihydropyridazines under various different conditions is described, leading to the formation of various products, including 2-aminopyrroles, phenylenediamines and varied novel heterocyclic motifs.

Key words 1,2-Dihydropyridazines, heterocycles, pyrroles, 1,2-diazetidines, phenylenediamines.

1,2-Dihydropyridazines 1 have rarely been employed in organic synthesis, despite their potential for the preparation of various heterocyclic motifs. Nevertheless, we recently demonstrated the application of these heterocycles in $4-\pi$-photocyclizations, generating bicyclic 1,2-diazetidines 2 that can be used to prepare a variety of novel molecular building blocks (Scheme 1). ${ }^{1}$ We also introduced a robust new preparation of 1,2-dihydropyridazines $\mathbf{1}$, which is suitable for use on multigram scale. ${ }^{2}$

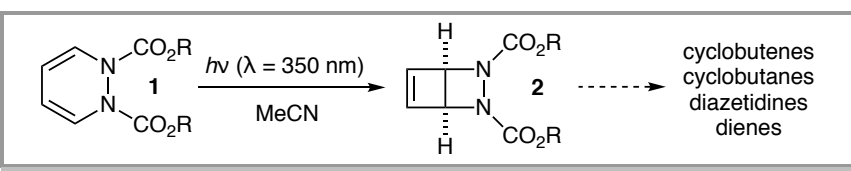

Scheme 14 - $\pi$-photocyclization of 1,2-dihydropyridazines 1 to give versatile bicyclic diazetidine products.

To further expand the applications of 1,2-dihydropyridazines, we studied their reactivity under a range of different conditions. Herein, we report the conversion of 1,2-dihydropyridazines into varied products, including phenylenediamine derivatives, 2aminopyrroles and various novel heterocyclic motifs.

Initially, we postulated that as a 1,3-diene, 1 might undergo DielsAlder reactions with suitable dienophiles to produce bicyclic hydrazine derivatives. However, attempted cycloaddition of $\mathbf{1}$ ( $\mathrm{R}$ $=\mathrm{Me}$, Et or $\mathrm{Pr}$ ) with reactive dienophiles (maleic anhydride, dimethyl acetylenedicarboxylate or benzoquinone) resulted only in recovered starting material, even upon heating at $100{ }^{\circ} \mathrm{C}$.
Conversely, reaction with 4-phenyl-1,2,4-triazole-3,5-dione (PTAD; an exceptionally reactive dienophile), led to fast conversion even at ambient temperature, but generated a complex mixture of products, and attempts to perform inverseelectron-demand Diels-Alder cycloadditions of $\mathbf{1}$ with a range of electron-rich dienophiles were also unsuccessful. The failure of these reactions is consistent with the findings of Fisher and coworkers, ${ }^{3}$ who reported that a similar dihydropyridazine bearing two phenyl substituents did not undergo Diels-Alder reaction with PTAD. This observation was attributed to the presumed high energy of the planar transition state that would be required for successful cycloaddition.

Interestingly, we observed that at temperatures above $100{ }^{\circ} \mathrm{C}$, dihydropyridazines $\mathbf{1}$ underwent isomerization into 2aminopyrroles 2 . The isomerization is proposed to proceed through initial $6-\pi$ electrocyclic ring opening to give diimines, which then undergo cyclization to give the corresponding 2aminopyrroles 2 (Scheme 2). Indeed, according to a computational study published by Lautens and Borden, such dihydropyridazines would be expected to undergo fast $6-\pi$ electrocyclic ring opening at elevated temperatures. ${ }^{4} \mathrm{~A}$ handful of examples of this dihydropyridazine-aminopyrrole isomerization have been reported to take place photochemically, ${ }^{1,5}$ but until now, the thermal promotion of this reaction has not been described. The optimal reaction conditions for the isomerization of $\mathbf{1}$ into $\mathbf{2}$ involved heating in xylene at $150^{\circ} \mathrm{C}$ for five hours, after which pyrroles 2 a-c were obtained in good to excellent yields (Scheme 2). ${ }^{6}$ Conversely, exposure of $\mathbf{1 d}$ to the same conditions resulted only in low yields of pyrrole $\mathbf{2 d}$, accompanied by small amounts of another pyrrole containing only one Boc group; the low yield of $2 \mathbf{d}$ can be explained by the instability of Boc groups at the high temperatures required for isomerization. In addition, ester-substituted dihydropyridazine $\mathbf{1 e}$ rearranged at slightly lower temperature $\left(130{ }^{\circ} \mathrm{C}\right.$ for five hours) to give disubstituted pyrrole 2e in 79\% yield. 2-Aminopyrroles 2a-e can be stored for several months under inert atmosphere in the freezer, but degrade rapidly within a few days under ambient conditions. 


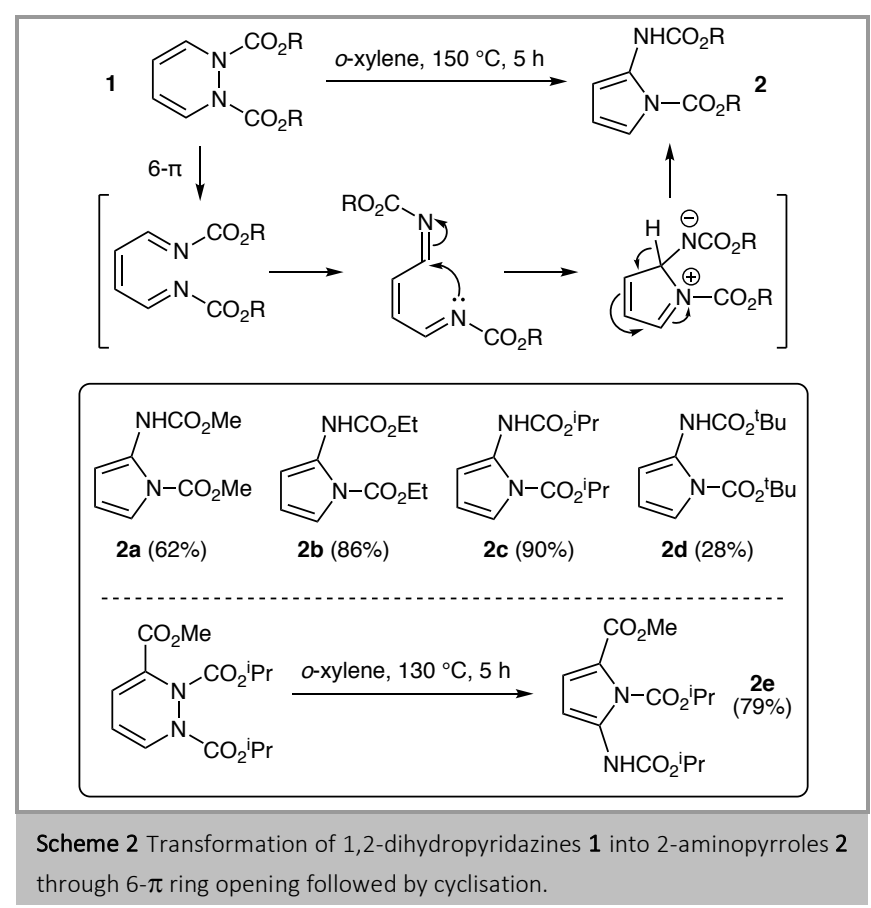

In contrast to the behaviour of 1,2-dihydropyridazine 1c, 2aminopyrrole $\mathbf{2 c}$ underwent facile reaction with reactive alkynes upon moderate heating, presumably generating Diels-Alder cycloadducts 3 , which would be expected to collapse to give substituted phenylenediamine derivatives 4 (Scheme 3). In this way, reaction of 1c with benzyne and dimethyl acetylenedicarboxylate furnished $\mathbf{4 a}$ and $\mathbf{4 b}$ respectively in good yields. However, less reactive alkynes (such as ethyl propiolate, ethyl phenylpropiolate and diphenylacetylene) failed to react with $2 \mathrm{c}$ even at elevated temperatures.

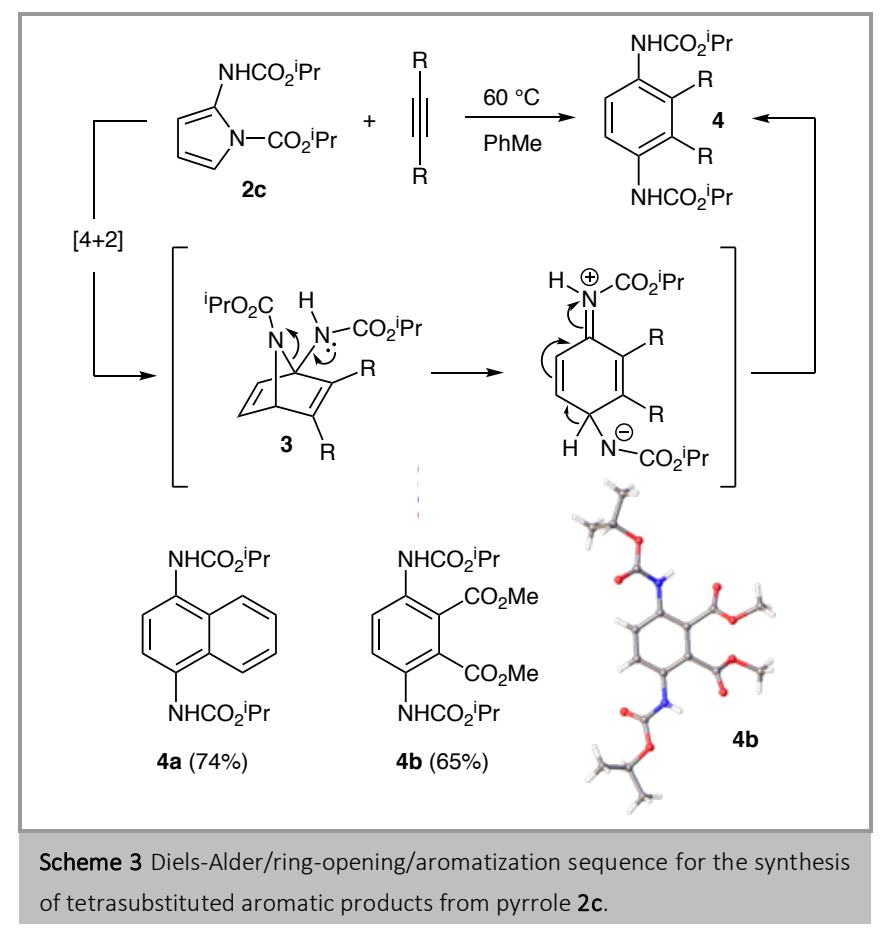

In related work, Arora and Mackay reported that a similar phenylenediamine formed upon heating of dihydropyridazine $\mathbf{1 b}$ with dimethyl acetylenedicarboxylate. ${ }^{7}$ The authors presumed that an initial Diels-Alder reaction occurred to give a bicyclic hydrazine derivative, which they stated "would aromatize readily", although no mechanism for this process was put forward. On the basis of our results, it seems much more likely that the 1,2-dihydropyridazine initially isomerized to the corresponding 2-aminopyrrole, which could then be transformed into the phenylenediamine product as outlined in Scheme 3.

The propensity of dihydropyridazine 1c to undergo common double bond functionalizations was also studied. The attempted mono-epoxidation of 1c using $m$-CPBA (meta-chloroperbenzoic acid) led to an interesting result - instead of the expected epoxide 5, diol trans-6 was obtained in 56\% yield, accompanied by recovered starting material and small amounts of other unidentifiable products (Scheme 4). The relative configurations of the hydroxyl groups were confirmed by X-ray diffraction, and trans- $\mathbf{6}$ is presumed to arise through initial epoxidation to produce 5, followed by epoxide opening under the acidic conditions, either through $\mathrm{S}_{\mathrm{N}} 2$ ' ring-opening by water, or mediated by the lone pair on one of the nitrogen atoms. In the latter case, the resulting iminium ion would then be trapped by water to generate diol trans-6 (Scheme 4).

$$
\text { 每) }
$$

Scheme 4 Attempted epoxidation of $1 \mathrm{c}$ using $m$-CPBA generates diol trans- 6 .

Similarly, the attempted aziridination of $\mathbf{1 c}$ using an iminoiodane reagent $(\mathrm{PhI}=\mathrm{NTs})$ in the presence of a copper catalyst did not lead to isolation of the expected aziridine 7. Complete conversion of the starting material was observed within 15 minutes, generating bis-sulfonamide trans-8 (Scheme 5), presumably through nucleophilic ring-opening of the putative aziridine intermediate $\mathbf{7}$ by toluenesulfonamide (likely generated as a degradation product of the aziridination reagent $\mathrm{PhI}=\mathrm{NTs}$ ) or by another derivative of PhI=NTs. Diamine trans-8 was isolated in good yield (62\%) as a single diastereoisomer, with the relative configurations of the sulfonamide groups being confirmed by $\mathrm{X}$ ray diffraction. Using just one equivalent of the iminoiodane reagent led to the same product, but in lower yield (38\%).

Dihydroxylation of $\mathbf{1 c}$ using osmium tetroxide did result in monofunctionalization as anticipated, although the resulting diol 9 was obtained as a mixture of two diastereoisomers (Scheme 6). The ${ }^{1} \mathrm{H} /{ }^{13} \mathrm{C}$ NMR spectra of both of the diol products were complex (as are those of the starting dihydropyridazines ${ }^{2}$ ), thus the diastereoisomer ratio in the crude product could not be determined directly by ${ }^{1} \mathrm{H}$ NMR spectroscopy. However, the diastereoisomers could be separated by flash column chromatography, resulting in a 2.7:1 ratio of the isolated major and minor diastereoisomers. Variable-temperature ${ }^{1} \mathrm{H} /{ }^{13} \mathrm{C}$ NMR experiments were also performed, but the signals were not fully 
resolved even upon heating at $75^{\circ} \mathrm{C}$, and NOESY experiments did not allow conclusive determination of the relative configurations of the hydroxyl groups in the two diastereoisomers. Potentially, diols $\mathbf{9}$ arise through ring-opening of the initial osmate ester by the lone pair on the adjacent nitrogen atom, generating an iminium ion that can be attacked by water to generate the diol products (Scheme 6). Interestingly, upon heating during NMR experiments, the minor diastereoisomer underwent slow conversion into the major diastereoisomer, although the reverse reaction was not observed. This epimerisation of the minor diatereoisomer into the major diastereoisomer starts to occur at $75{ }^{\circ} \mathrm{C}$ in $\mathrm{d}_{6}$-DMSO, and takes place more rapidly at higher temperatures.

$\mathrm{Phl}=\mathrm{NTs}(2.0 \mathrm{eq}$.
Scheme 5 Attempted aziridination of $1 \mathrm{c}$ using Phl=NTs generates diamine
trans-8.

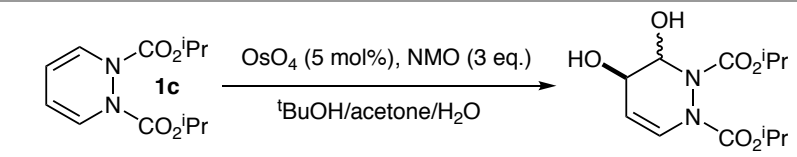

$$
\begin{aligned}
& 9 \text { (78\%; 2.7:1 dr) }
\end{aligned}
$$

Scheme 6 Dihydroxylation of $1 \mathrm{c}$ using osmium tetroxide and $N$-methyl morpholine $\mathrm{N}$-oxide (NMO)

Finally, the reaction of $\mathbf{1 c}$ with in situ-generated dichlorocarbene resulted in the dichlorocyclopropanation of both double bonds to give trans-10 in 71\% yield (Scheme 7), with the relative configurations of the cyclopropane rings being confirmed by $\mathrm{X}$ ray diffraction. Tricycle trans-10 displayed surprising thermal stability, as it remained unchanged after heating at reflux in xylenes for six hours.

In conclusion, this preliminary study explored the applications of 1,2-dihydropyridazines, allowing the preparation of a wide range of different products (e.g. 2-aminopyrroles, phenylenediamines and other novel heterocycles). The products obtained from manipulations of the 1,2-dihydropyridazines were often unexpected, but now that the fundamental reactivity patterns of these under-exploited heterocycles have been established, application to more complex 1,2-dihydropyrdazines will allow access to an even broader range of novel products.

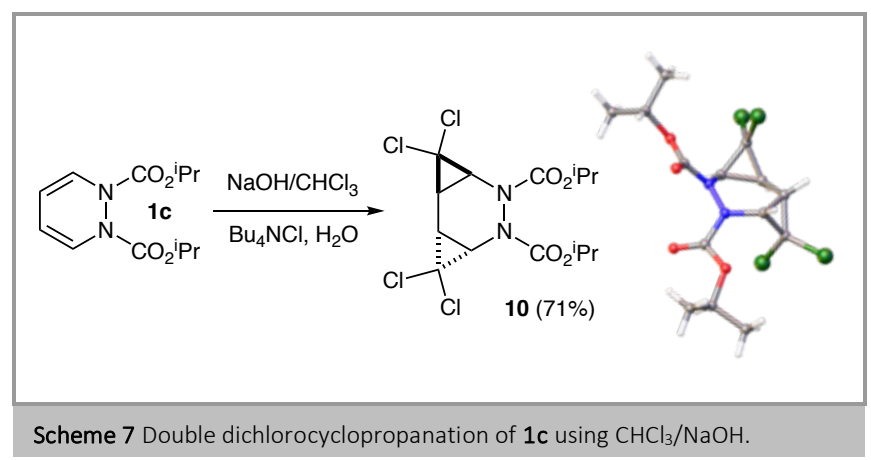

\section{Funding Information}

Funding from AstraZeneca (CASE Top-Up Award to T.K.B.; 2015-2019) is gratefully acknowledged.

\section{Supporting Information}

YES

\section{Primary Data}

NO

\section{References and Notes}

(1) Britten, T. K.; Kemmitt, P. D.; Halcovitch, N. R.; Coote, S. C. Org. Lett. 2019, 22, 9232.

(2) Britten, T. K.; Akien, G. R.; Kemmitt, P. D.; Halcovitch, N. R.; Coote, S. C. Tetrahedron Lett. 2019, 60, 1497.

(3) Fisher, T. H.; Crook, J. C.; Chang, S. Tetrahedron 1987, 43, 2443.

(4) Chen, B.; Scott, M. E.; Adams, B.; Hrovat, D.; Borden, W. T.; Lautens, M. Org. Lett. 2014, 16, 3930.

(5) (a) Altman, L. J.; Semmelhack, M. F.; Hornby, R. B.; Vederas, J. C. Chem. Commun. 1968, 686; (b) Sheradsky, T.; Moshenberg, R. J. Org. Chem. 1984, 49, 587; (c) Sheradsky, T.; Moshenberg, R. J. Org. Chem. 1985, 50, 5604.

(6) Representative procedure for the thermal isomerization of 1,2dihydropyridazines into 2-aminopyrroles: A solution of 1,2dihydropyridazine 1a (520 mg, $2.62 \mathrm{mmol}, 1.0 \mathrm{eq}$ ) in $o$-xylene (5 $\mathrm{mL}$ ) was heated at reflux for 5 hours under argon. Purification by flash column chromatography on silica gel (eluent: hexane-EtOAc, $100 \%$ hexane $\rightarrow 9: 1$ ) gave 2-aminopyrrole 2 a $(320 \mathrm{mg}, 1.61 \mathrm{mmol}$, $62 \%$ ) as a white solid. Note: 2-Aminopyrroles must be stored in the freezer under an inert atmosphere in order to prevent degradation. $R_{f}$ (Hexane-EtOAc, $\left.2: 1\right)=0.35 ;$ m.p. $=46-47^{\circ} \mathrm{C} ;{ }^{1} \mathrm{H} \mathrm{NMR}(400 \mathrm{MHz}$, $\mathrm{CDCl}_{3}$ ); $\delta 9.05$ (br s, $\left.1 \mathrm{H}, \mathrm{NH}\right), 6.85$ (dd, $J=3.6,1.8 \mathrm{~Hz}, 1 \mathrm{H}, \mathrm{Ar}-\mathrm{H}$ ), 6.42-6.32 (br m, 1H, Ar-H), $6.13(\mathrm{t}, J=3.6 \mathrm{~Hz}, 1 \mathrm{H}, \mathrm{Ar}-\mathrm{H}), 3.95(\mathrm{~s}$, $3 \mathrm{H}, \mathrm{OMe}), 3.77$ (s, 3H, OMe); ${ }^{13} \mathrm{C}$ NMR $\left(100 \mathrm{MHz}, \mathrm{CDCl}_{3}\right) ; \delta 153.1$ $(\mathrm{C}=0), 152.4(\mathrm{C}=0), 130.5(\mathrm{Ar}-\mathrm{C}), 114.0(\mathrm{Ar}-\mathrm{CH}), 111.6(\mathrm{Ar}-\mathrm{CH})$, 98.4 (Ar-CH), 54.2 (OMe), $52.6(\mathrm{OMe})$; FTIR (ATR) $v\left(\mathrm{~cm}^{-1}\right): 3349$ $(\mathrm{NH}), 2950,1724(\mathrm{C}=0)$; HRMS (APCI): $m / z$ calculated for: $\mathrm{C}_{8} \mathrm{H}_{10} \mathrm{~N}_{2} \mathrm{O}_{4}[\mathrm{M}+\mathrm{H}]^{+}$199.0713, found 199.0712.

(7) Arora, P. C.; Mackay, D. J. Chem. Soc. D Chem. Commun. 1969, 677. 